\title{
Radio Frequency-Activated Nanoliposomes for Controlled Combination Drug Delivery
}

\author{
Swapnil A. Malekar, ${ }^{1}$ Ashish L. Sarode, ${ }^{1}$ Alvin C. BachII, ${ }^{1}$ Arijit Bose, ${ }^{2}$ \\ Geoffrey Bothun, ${ }^{2}$ and David R. Worthen ${ }^{1,2,3}$
}

Received 27 February 2015; accepted 12 April 2015; published online 22 April 2015

\begin{abstract}
This work was conducted in order to design, characterize, and evaluate stable liposomes containing the hydrophobic drug raloxifene $\mathrm{HCl}$ (RAL) and hydrophilic doxycycline $\mathrm{HCl}$ (DOX), two potentially synergistic agents for treating osteoporosis and other bone lesions, in conjunction with a radio frequency-induced, hydrophobic magnetic nanoparticle-dependent triggering mechanism for drug release. Both drugs were successfully incorporated into liposomes by lipid film hydration, although combination drug loading compromised liposome stability. Liposome stability was improved by reducing the drug load and by including Pluronics ${ }^{\circledR}$ (PL) in the formulations. DOX did not appear to interact with the phospholipid membranes comprising the liposomes, and its release was maximized in the presence of radio frequency (RF) heating. In contrast, differential scanning calorimetry (DSC) and phosphorus-31 nuclear magnetic resonance $\left({ }^{31} \mathrm{P}-\mathrm{NMR}\right)$ analysis revealed that RAL developed strong interactions with the phospholipid membranes, most notably with lipid phosphate head groups, resulting in significant changes in membrane thermodynamics. Likewise, RAL release from liposomes was minimal, even in the presence of RF heating. These studies may offer useful insights into the design and optimization of multidrug containing liposomes. The effects of RAL on liposome characteristics and drug release performance underscore the importance of appropriate physical-chemical analysis in order to identify and characterize drug-lipid interactions that may profoundly affect liposome properties and performance early in the formulation development process.
\end{abstract}

KEY WORDS: controlled release; drug combination; liposomes; nanoparticles.

\section{INTRODUCTION}

Drug therapy is routinely employed for palliating osteoporosis progression and for treating bone fractures in osteoporosis-weakened bones. Raloxifene $\mathrm{HCl}$ (RAL), a systemic selective estrogen receptor modulator that exerts tissue-specific estrogenic action on bone tissue, is approved for osteoporosis therapy, although its low oral bioavailability (ca. $2 \%$ ) and untoward side effects, including hot flashes, cramping, teratogenicity, and potentially lethal blood clot formation, limit its utility as an orally administered systemic drug (1,2). Given these biopharmaceutical and therapeutic liabilities, alternative formulations, including transdermal nanopolymersomes, have been explored as alternative systems for RAL delivery $(2,3)$. Doxycycline $\mathrm{HCl}$ (DOX), a tetracycline antibiotic with anti-rheumatic and anti-inflammatory properties, inhibits

\footnotetext{
${ }^{1}$ Department of Biomedical and Pharmaceutical Sciences, College of Pharmacy, University of Rhode Island, Kingston, Rhode Island 02881, USA.

${ }^{2}$ Department of Chemical Engineering, College of Engineering, University of Rhode Island, 495M Pharmacy Building, 7 Greenhouse Drive, Kingston, Rhode Island 02881, USA.

${ }^{3}$ To whom correspondence should be addressed. (e-mail: drworthen@uri.edu)
}

metalloproteinase activity and bone resorption and has been employed for the treatment of arthritis and for its bone healing properties, although its oral systemic use is often associated with nausea and skin disorders. DOX is contraindicated in pregnancy and lactation due to untoward effects on tooth and bone formation (4). Given their synergistic potential, controlled release RAL/DOX combination therapy targeted to affected skeletal tissues such as osteoporotic lesions and bone fractures may represent a novel and attractive option for localized bone therapy. Despite its therapeutic potential, RAL/DOX combination therapy has yet to be explored, and prototype formulations for their localized, controlled release have yet to be evaluated. Given the growing interest in intratrabecular and other modes of direct drug delivery to bone, the successful development of controlled release, combination, controlled release RAL/DOX formulations might be particularly useful for the treatment of isolated bone lesions $(5,6)$.

Liposomes and other lipid-based vesicular systems may serve as useful drug delivery systems for various therapeutic agents (7). Hydrophilic agents may be encapsulated in the aqueous cores of the liposomes, while hydrophobic compounds may be incorporated into the lipid bilayers (3). The stability of liposomes is always a major concern, as poor stability may lead to vesicular aggregation and fusion, liposomal cargo leakage, degradation, and compromised product 
stability and drug release at desired target sites (8). Various attempts have been made to stabilize liposomes, such as including cholesterol in the lipid bilayers in order to increase bilayer rigidity, the use of phospholipids of high transition temperatures, polymerization of modified lipids, freeze-thawing, and the insertion of block copolymers (9-13). The stability of liposomes may be enhanced by steric hindrance of aggregation with the aid of poloxamer block copolymers (Pluronics ${ }^{\circledR}(\mathrm{PL})$ ). As integral parts of liposomal membranes, PLs may enhance liposomal stability for an extended period of time $(14,15)$.

Since the release of hydrophilic drugs encapsulated in the liposomal core is typically driven by passive diffusion, the insertion of stabilizing PL chains into the bilayers may hinder this process (16-18). The enhanced rigidity of the bilayer that could be imparted by the inclusion of hydrophobic drugs might likewise stabilize the systems and reduce the drug release rate. Moreover, the release of hydrophobic compounds embedded within the lipid bilayers could be compromised due to strong hydrophobic interactions that might develop between the drugs and the lipid chains. A high-frequency alternating current (AC) magnetic field has been reported to trigger drug release from liposomes and polymersomes with the use of super paramagnetic iron oxide (SPIO) nanoparticles (19-22). These SPIOs are biocompatible with minimal in vivo toxicity and have also been used as diagnostic agents $(21,22)$. In this investigation, it was hypothesized that RAL and DOX could be successfully incorporated into sterically stabilized dipalmitoylphosphatidyl choline (DPPC)-based liposomes produced with the aid of a triblock copolymer for stabilization in conjunction with the use of SPIO and radio frequency magnetic fields to trigger the release of the drugs. It was further hypothesized that DOX, a highly water soluble drug, would be encapsulated into the hydrophilic cores of the liposomes, whereas RAL, with much lower water solubility, would be localized in the lipid bilayers. Extensive characterization of the liposome formulations and their stability were performed, and both differential scanning calorimetry (DSC) and phosphorus-31 nuclear magnetic resonance $\left({ }^{31} \mathrm{P}-\mathrm{NMR}\right)$ studies were performed in order to better understand the notable and previously unreported effects of the incorporated drugs, in particular those imparted by RAL, on DPPC-based liposome stability, physical-chemical properties, and drug release.

\section{MATERIALS AND METHODS}

\section{Materials}

1,2-Dipalmitoyl-sn-glycero-3-phosphocholine monohydrate (DPPC) was purchased from Corden Pharma (CO, USA). Doxycycline hydrochloride (DOX) and raloxifene hydrochloride (RAL) were purchased from Fisher Scientific (Pittsburgh, PA). All Pluronic $®$ polymers were purchased from BASF (Parsippany, NJ). Cellulose membranes (Spectra/Por, MW cutoff $3500 \mathrm{Da}$ ), used for dialysis and drug release tests, were obtained from Spectrum Laboratories, Inc. (Houston, TX). Phosphate-buffered saline (PBS) tablets were purchased from MP Biomedicals (Solon, OH). SPIO maghemite nanoparticles $\left(5 \mathrm{~nm}, 24 \mathrm{mg} \mathrm{mL}^{-1}\right.$, or $187.9 \mathrm{mM}$ $\mathrm{Fe}_{2} \mathrm{O}_{3}$ ) dispersed in chloroform were purchased from Ocean Nanotech (Springdale, AR). On the basis of the density of maghemite $\left(4.9 \mathrm{~g} \mathrm{~cm}^{-3}\right), 24 \mathrm{mg} \mathrm{mL}^{-1}$ is equivalent to $1.43 \times 10^{17}$ particles per milliliter. All other reagents were purchased from Fisher Scientific and were of analytical grade.

\section{Liposome Preparation}

Vesicles were prepared at a $17-\mathrm{mM}$ lipid concentration for all formulations. The vesicles were prepared in 4-mL batches by film rehydration $(3 \mathrm{~mL}$ for dialysis and drug release studies and $1 \mathrm{~mL}$ for characterization) as described by Chen et al. (22). The samples were further diluted to a lipid concentration of $5.6 \mathrm{mM}$ for TEM, $1 \mathrm{mM}$ for dynamic light scattering (DLS) and zeta potential analysis, and $0.1 \mathrm{mM}$ for nano-DSC analysis. When individual drugs were loaded into liposomes, their final concentration was maintained at $2 \mathrm{mM}$. When the two-drug combination was encapsulated, their concentrations were reduced to $0.5 \mathrm{mM}$, as higher two-drug concentrations resulted in vesicular aggregation and instability. The procedure was the same for all of the formulations except for the step in which various components were added for different formulations. DOX- containing liposomes were prepared by dissolving $50 \mathrm{mg}$ of DPPC in $4 \mathrm{~mL}$ of chloroform. The chloroform was removed by rotary evaporation at $50^{\circ} \mathrm{C}$ (above the DPPC melting temperature) starting at 450 mbar for $30 \mathrm{~min}$, then decreased to $300 \mathrm{mbar}$ for $30 \mathrm{~min}$, and finally $200 \mathrm{mbar}$ for $30 \mathrm{~min}$. This lipid film was kept under vacuum for $2 \mathrm{~h}$ at room temperature in order to remove traces of chloroform. The film was then rehydrated with a $2-\mathrm{mM}$ solution of DOX dissolved in $137 \mathrm{mM}$ PBS for $2 \mathrm{~h}$ at $50^{\circ} \mathrm{C}$. RAL liposomes were analogously prepared. RAL and DPPC were dissolved in a 3:1 ratio of chloroform/methanol due to the insolubility of RAL in pure chloroform. The organic solvents were removed by rotary evaporation at $50^{\circ} \mathrm{C}$ (above the DPPC melting temperature) starting at $450 \mathrm{mbar}$ for $30 \mathrm{~min}$, then decreased to $300 \mathrm{mbar}$ for $30 \mathrm{~min}$, and finally $200 \mathrm{mbar}$ for $30 \mathrm{~min}$. This lipid film was kept under vacuum for $2 \mathrm{~h}$ at room temperature to remove traces of organic solvents. The film was then rehydrated with $137 \mathrm{mM}$ PBS, and the final RAL concentration in the formulation was $2 \mathrm{mM}$. The magnetic nanoparticles (MNPs), RAL, DOX, and Pluronic $\AA$ containing liposomes were prepared in a similar way by adding the MNPs [lipid/MNP (L/N) ratios of 5000:1, 10,000:1, and 20,000:1] and P84 to the organic solvent mixture containing lipid and RAL, and following the film formation as described above using rotary evaporator, the film was rehydrated with $0.5 \mathrm{mM}$ DOX in $137 \mathrm{mM}$ PBS. The resulting aqueous dispersions were then sonicated for $1 \mathrm{~h}$ at room temperature using a bath sonicator. For the ${ }^{31} \mathrm{P}-\mathrm{NMR}$ studies, the vesicles were analogously prepared, with the aqueous phase containing $10 \% v / v \mathrm{D}_{2} \mathrm{O}$ for a lock signal.

\section{Cryogenic Transmission Electron Microscopy}

Cryogenic transmission electron microscopy (CryoTEM) samples were prepared at $37^{\circ} \mathrm{C}$ using a Vitrobot (FEI Company), a PC-controlled robot for sample vitrification. Quantifoil grids were used with 2- $\mu \mathrm{m}$ carbon holes on 200 square mesh copper grids (Electron Microscopy Sciences, Hatfield, PA). After immersing the grid into the sample, it was then removed, blotted to reduce film thickness, and vitrified in liquid ethane. Imaging was performed in a cooled microscopy stage (Model 915, Gatan Inc., Pleasanton, CA) at $200 \mathrm{kV}$ using a JEOL JEM-2100F TEM (Peabody, MA). 


\section{Energy-Dispersive X-ray Scattering}

Energy-dispersive X-ray scattering (EDS) (Model INCAx-act, Oxford Instrument, K) was used to detect elemental iron from the magnetic nanoparticles within the iron oxide nanoparticle-loaded liposomes. EDS was conducted during cryogenic imaging with $158 \mathrm{~s}$ of live time and $92 \mathrm{~s}$ of dead time.

\section{Phosphorus-31 Nuclear Magnetic Resonance Spectroscopy}

The ${ }^{31} \mathrm{P}$-NMR spectra were acquired on an Agilent NMRS 500 NMR spectrometer operating at $202.3 \mathrm{MHz}$ using a 5-mm OneNMR probe. The probe temperature was thermostated at $37^{\circ} \mathrm{C}$ for all experiments. Liposome formulations analyzed by NMR were prepared as previously described with the exception that $10 \% \mathrm{D}_{2} \mathrm{O}$ was used as a solvent in order to provide a deuterium lock signal. NMR data were collected for $60 \mathrm{~K}$ scans with a $35.7-\mathrm{kHz}$ sweep width using $131 \mathrm{~K}$ data points. Acquisition time was $1.3 \mathrm{~s}$ with a relaxation delay of $0.5 \mathrm{~s}$. The data were processed with Mnova program V8.1 Mesterlab research SL. A line broadening of $50 \mathrm{~Hz}$ was applied to all spectra. All spectra were indirectly referenced to $\mathrm{H}_{3} \mathrm{PO}_{4}$ set to $0 \mathrm{ppm}$. Data were acquired without spinning.

\section{Nanodifferential Scanning Calorimetry}

Nanodifferential scanning calorimetry (nano-DSC) was performed using a TA Instruments Nano DSC (New Castle, DE, USA). Samples at a concentration of $0.1 \mathrm{mM}$ lipid were degassed under vacuum for $30 \mathrm{~min}$ before loading into a 0.6-mL capillary cell. The cell was then pressurized with nitrogen to $1 \mathrm{~atm}$ and equilibrated at $25^{\circ} \mathrm{C}$. The sample was scanned at $1^{\circ} \mathrm{C} \min ^{-1}$ over a range of 25 to $60^{\circ} \mathrm{C}$.

\section{High-Performance Liquid Chromatography (Employed for Drug Stability Confirmation and Quantification of Drug Release)}

The high-performance liquid chromatography (HPLC) system comprised a Hitachi LaChrome Elite equipped with a PDA detector and an automatic injector with a loop volume of $0.1 \mathrm{~mL}$. For DOX quantification, an Agilent Zorbax SB C8 $(5 \mu \mathrm{m}, 4.6 \times 250 \mathrm{~mm})$ column was used. The mobile phase consisted of $0.02 \mathrm{M}$ oxalic acid/methanol/acetonitrile $(65 / 25 / 10)$ with a final $\mathrm{pH}$ of 2.5 . The flow rate was $1 \mathrm{~mL} \mathrm{~min}^{-1}$ with an injection volume of $90 \mu \mathrm{L}$ and a detection wavelength of $350 \mathrm{~nm}$. The limit of detection of DOX using this method was $20 \mathrm{nM}$. The calibration curve in PBS had an $R^{2}=0.9999$. The column used for RAL quantification was a Luna $3 \mu \mathrm{m} \mathrm{C18} \mathrm{(2)} 150 \times 4.6 \mathrm{~mm}$ with a mobile phase comprising $0.05 \mathrm{M}$ ammonium acetate/acetonitrile (67/33) with a final $\mathrm{pH}$ of 4.0. The flow rate was $1 \mathrm{~mL} \mathrm{~min}^{-1}$ with an injection volume of $90 \mu \mathrm{L}$ and a detection wavelength of $287 \mathrm{~nm}$. The limit of detection was $20 \mathrm{nM}$, and the calibration curve in PBS had an $R^{2}=0.9997$. $R^{2}$ value of $0.9997-9$ over a range of 20 to $200,000 \mathrm{nM}$ suggests accurate quantification of drug derived from corresponding HPLC chromatograms.

\section{Dynamic Light Scattering}

DLS measurements were performed using a Malvern Instruments Zetasizer Nano ZS with a backscattering detector angle of $173^{\circ}$ and a 4-mW, 633-nm He-Ne laser (Worcestershire, UK). For size distribution studies, $1 \mathrm{~mL}$ of the liposome formulations was analyzed in an optical grade polystyrene cuvette at $37^{\circ} \mathrm{C}$. Before analysis, the samples were stored at $37^{\circ} \mathrm{C}$ and then analyzed after $24 \mathrm{~h}$.

\section{Radio Frequency-Induced Drug Release}

Drug-loaded liposomes containing magnetic nanoparticles were placed in a copper heating coil (three turns at $4.5 \mathrm{~cm}$ mean diameter) around a custom-designed polycarbonate container with a holder for SpectraPor dialysis tubing. Heating was conducted as a function of time and electromagnetic field strength using a 1-kW Hotshot (Ameritherm Inc., Scottsville, NY) operating up to $250 \mathrm{~A}$ and $281 \mathrm{kHz}$. Samples were collected from the drug dissolution media at serial time points during the drug release experiments, and drug concentrations as a function of time were then determined using HPLC.

\section{Dialysis and Release Studies}

The dialysis experiments were conducted at room temperature $\left(25 \pm 0.5^{\circ} \mathrm{C}\right)$ using $3.5-\mathrm{kDa}$ tubular cellulose acetate membranes for $24 \mathrm{~h}$ in $137 \mathrm{mM}$ PBS with constant stirring and replacement of the dissolution media (22). The dissolution media was collected and analyzed by HPLC for unencapsulated drug in order to calculate the drug loading capacity of the liposomes. Drug release studies were performed using the same dialysis tubing. The experiments were carried out in $137 \mathrm{mM}$ PBS at $37 \pm 0.5^{\circ} \mathrm{C}$ and a $\mathrm{pH}$ of 7.4 with a stirring speed of $75 \mathrm{rpm}$ using a 0.5 -in. magnetic stirrer. Fresh media was replaced after the sampling was done at regular time intervals.

\section{RESULTS}

\section{Morphological Characterization of the Vesicles}

The structural characteristics of the liposomes, including size and shape, were assessed upon the incorporation of P84, MNPs, DOX, and RAL and compared to bare DPPC liposome controls. In order to study the morphological characteristics and incorporation of MNPs into the bilayer, Cryo-TEM and EDS analyses of the liposomes were performed. The morphological characteristics of liposomal formulations containing DOX or RAL at $2-\mathrm{mM}$ individual drug concentrations are shown in Fig. 1a, b. Both DOX- and RAL-containing liposomes were similar in shape, suggesting that the drugs alone did not influence liposomal morphology. The formulations containing both P84 and MNPs were also analyzed for their morphology and elemental composition (Fig. 2).

As expected, formulations that did not contain MNPs did not show any iron peak, as seen in Fig. 2b. Formulations containing MNPs (Fig. 2d) depicted distinct iron peaks at $6.4 \mathrm{KeV}$. There was no evidence of the presence of MNPs or aggregates of MNPs outside of the liposomes. The EDS scans were taken in the specific areas of the grid that had liposomes and no prevalent 


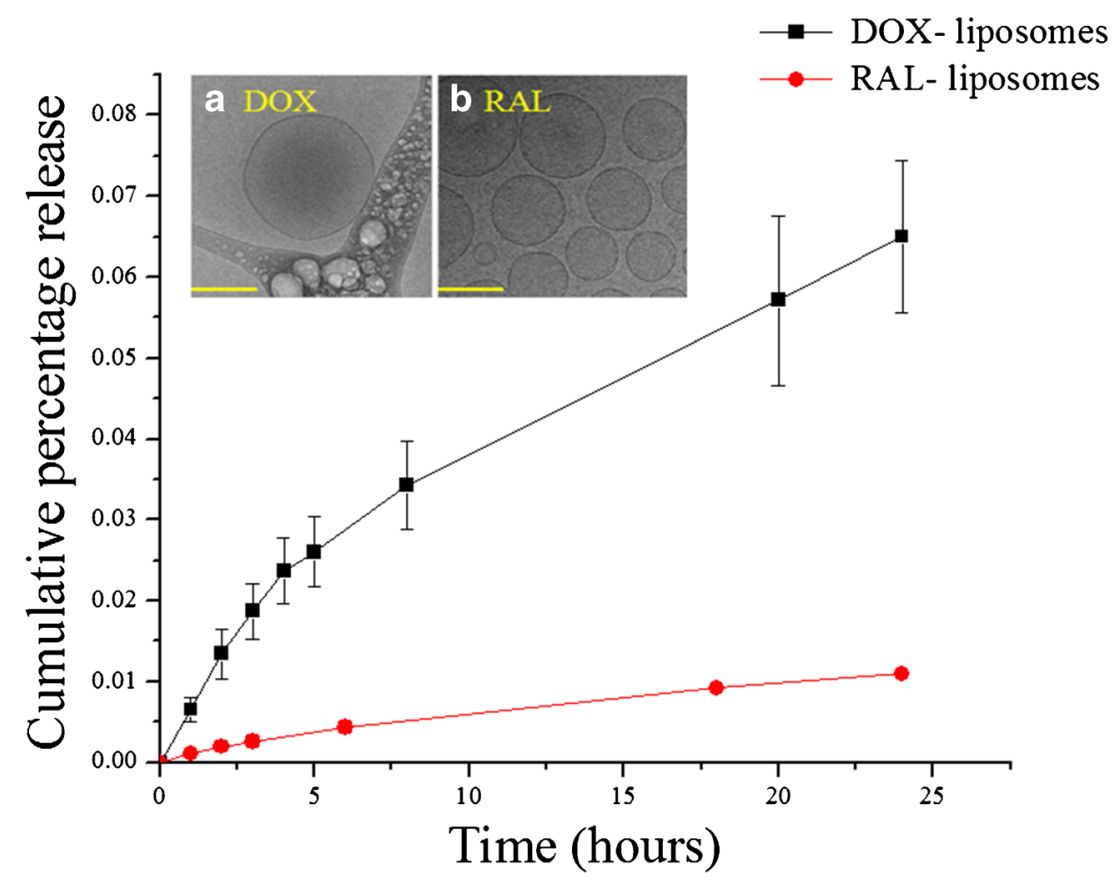

Fig. 1. DOX and RAL release from DPPC liposomes carried out at $37^{\circ} \mathrm{C}$ and $\mathrm{pH} 7.4$ in $137 \mathrm{mM}$ PBS. $1.88 \mathrm{mM}$ of DOX and $1.98 \mathrm{mM}$ RAL were encapsulated after $20 \mathrm{~h}$ of dialysis which corresponds to encapsulation efficiency of 94.5 and $99.3 \%$ respectively. Cryo-TEM images of corresponding a DOX and $\mathbf{b}$ RAL containing liposomes indicating no difference in the morphology. Scale bar is $200 \mathrm{~nm}$ for the TEM images

iron oxide nanoparticles. This suggests that iron oxide nanoparticles were successfully incorporated into the bilayers.

\section{Stability of Liposomes}

The polydispersity indices (PDI) of the liposomal formulations were obtained from photon correlation spectroscopic analysis, giving a dimensionless number extrapolated from the autocorrelation function. The PDI of all of the liposome formulations assessed in the present study was below 0.3. The liposomal formulations were further characterized by DLS in order to estimate their stability at physiologic temperature (Fig. 3).

By taking into consideration the goal of multiple drug loading, when $2 \mathrm{mM}$ of both DOX and RAL were loaded into analogous liposomal systems, large aggregates formed within a few hours. In order to prepare stable liposomes containing both drugs, their concentration was reduced from 2 to $0.5 \mathrm{mM}$ each. The reduction in drug concentration did not improve liposomal stability. Since one goal of this study was to make stabilized nanoliposomes, poly (ethylene oxide)-poly (propylene oxide)-poly (ethylene oxide) triblock copolymers (PL) were incorporated into the bilayers in order to enhance vesicular stability. Pluronics ${ }^{\circledR}$ F-127 and F-108 have been shown to increase the mechanical stability of some lipid-based vesicular systems, such as dioleoylphosphatidylethanolamine liposomes (23). Therefore, various grades of PLs were incorporated at different weight percentages into the liposomal bilayers in order to optimize the stability of liposomes containing $0.5 \mathrm{mM}$ of both DOX and RAL (Table I). Pluronic P84 was the only grade of this polymer that afforded increased stability in these systems.

\section{Bilayer Phase Characterization}

The change in the transition temperature of a vesicular lipid bilayer depends upon the presence of other chemical species and their subsequent interaction within the different domains of the vesicles. The interactions of DOX, RAL, and combinations thereof were thermally analyzed using nano-DSC (Fig. 4). For drug-free DPPC control liposomes, a transition of $8.43 \mathrm{Kcal} \mathrm{mol}^{-1}$ occurred at $42.96^{\circ} \mathrm{C}$ (Table II). This transition is consistent with the conversion of the rippled gel phase to the liquid crystalline phase (24). Upon the addition of $2 \mathrm{mM}$ RAL to the liposome formulation, a significant phase transition of $12.12 \mathrm{Kcal} \mathrm{mol}^{-1}$ at $49.24^{\circ} \mathrm{C}$ was observed. The addition of $0.5 \mathrm{mM}$ RAL showed a transition of $10.98 \mathrm{Kcal} \mathrm{mol}^{-1}$ at $47.5^{\circ} \mathrm{C}$. The inclusion of MNPs (L/N ratio of $10,000 / 1)$ decreased the $T_{\mathrm{m}}$ significantly to $39.03^{\circ} \mathrm{C}$.

The effect of incorporating DOX, RAL, and P84 in DPPC liposomes was also examined by ${ }^{31} \mathrm{P}-\mathrm{NMR}$, as shown in Fig. 5. Blank DPPC liposomes displayed one single sharp peak, a spectrum characteristic of small, unilamellar vesicles (25). Upon the addition of either DOX or P84 to DPPC liposomes, neither a chemical shift nor a change in the shape of the NMR signal was detected. The presence of small, unilamellar vesicles was also confirmed with cryo-TEM imaging. Thus, it was concluded that neither DOX nor P84 affected the orientation or the environment of the phosphate head groups in the liposomal bilayers. In contrast, upon the incorporation of RAL, an additional resonance (indicated with black arrows) appears upfield of DPPC, which is indicative of increased exposure of the phosphate head groups at the surface of the membranes. 

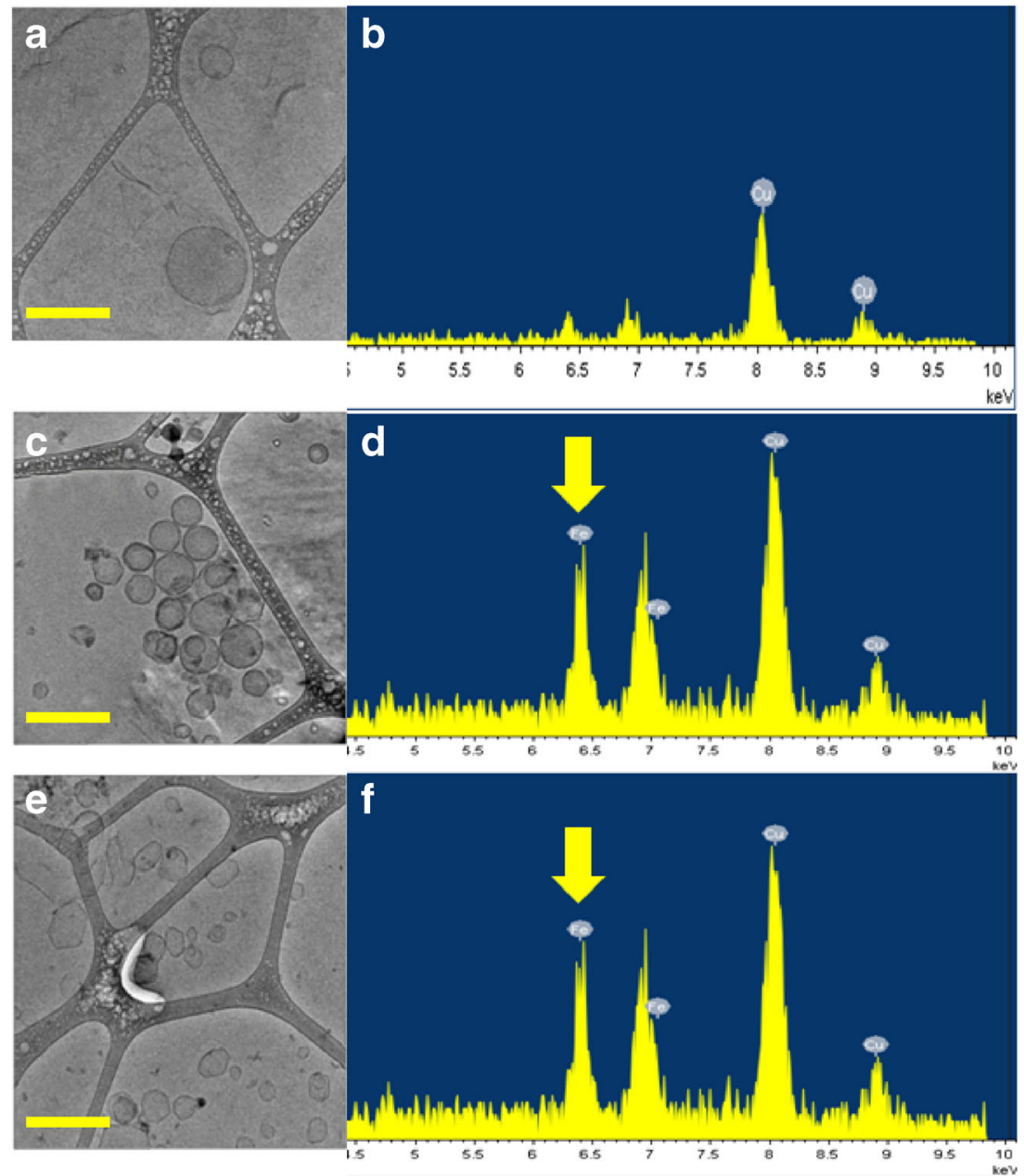

Fig. 2. Cryo-TEM images (a) and EDS scans (b) of liposomes containing DOX, RAL, and P84 which show absence of iron peak at $6.4 \mathrm{keV}$. Cryo-TEM images and EDS scans of liposomes containing DOX, RAL, P84, and MNPs before (c, d) and after (e, f) RF exposure. The $\mathrm{L} / \mathrm{N}$ ratio was 10,000:1 for these formulations. Yellow arrows indicate the presence of MNPs in the bilayers. The round shape of liposomes was converted to angular showing the influence of RF heating on the bilayers. Scale bar is $200 \mathrm{~nm}$

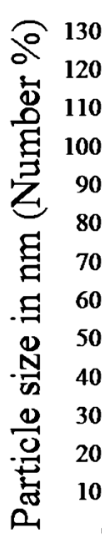

1) $2 \mathrm{mM} D O X+2 \mathrm{mM} R A L$

2) $0.5 \mathrm{mM}$ DOX $+0.5 \mathrm{mM}$ RAL

3) $2 \mathrm{mM}$ RAL

4) $0.5 \mathrm{mM}$ DOX + 0.5mM RAL + P84 + MNP

5) $2 \mathrm{mM} \mathrm{DOX}$

6) $0.5 \mathrm{mM} \mathrm{DOX}+0.5 \mathrm{mMRAL}+\mathrm{P} 84$ 


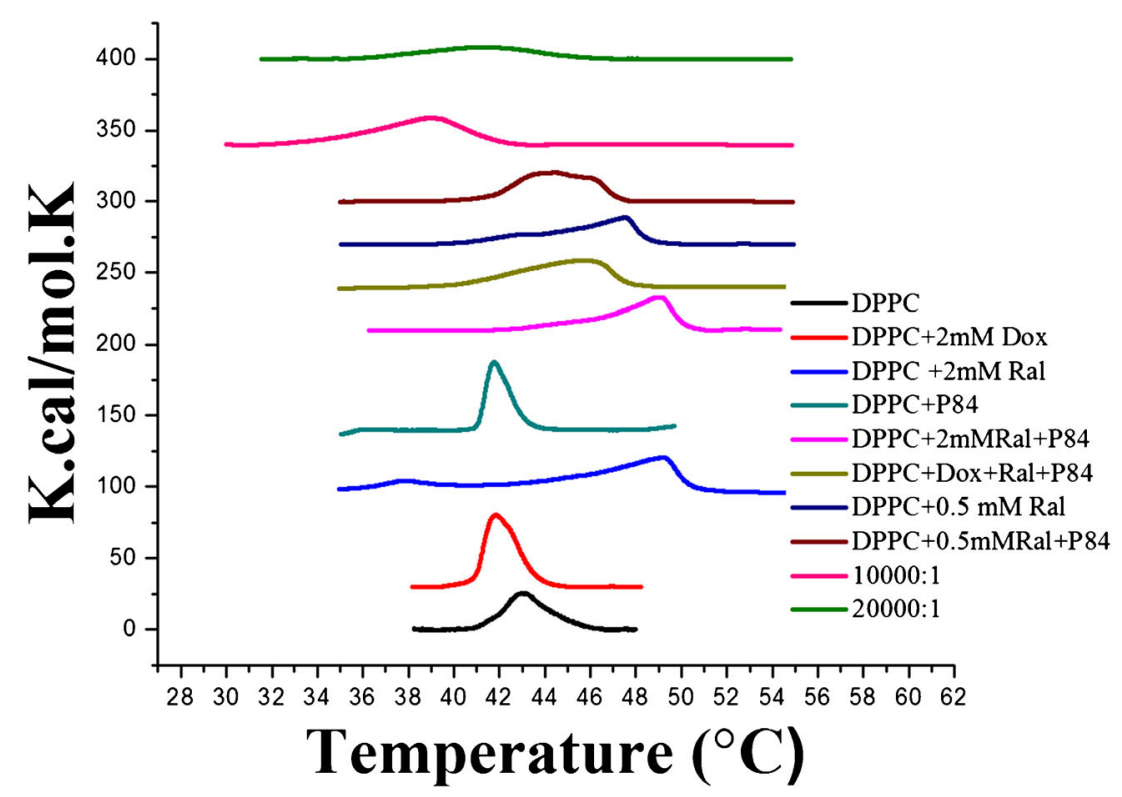

Fig. 4. Nano-DSC thermographs of the liposomal formulations. RAL increased the $T_{\mathrm{m}}$ of the liposomal bilayer, whereas the $T_{\mathrm{m}}$ was reduced using MNPs and peak broadening was observed with P84. DOX and P84 did not have any effect on the $T_{\mathrm{m}}$

The dialysis experiment was carried out until a constant drug concentration was obtained from assayed dialysate samples, indicating that equilibrium had been achieved and no unentrapped material was present in the formulation. At the end of the dialysis experiment, mass balance equations were used to calculate the encapsulation efficiency of the liposomal formulations. At a 17-mM DPPC concentration, $1.88 \mathrm{mM}$ (94.56\% theoretical) of DOX and $1.98 \mathrm{mM}$ (99.35\% theoretical) of RAL were successfully encapsulated. While performing individual drug release studies, it was observed that DOX entrapped in the hydrophilic core was released

Table II. Enthalpy, Entropy, and the Transition Temperatures of Liposomal Formulations Measured by Nano-DSC

\begin{tabular}{|c|c|c|c|}
\hline Formulation & $\begin{array}{l}\Delta H \\
(\mathrm{Kcal} / \mathrm{mol})\end{array}$ & $\begin{array}{l}\Delta S \\
{[\mathrm{Kcal} /(\mathrm{mol} . \mathrm{K})]}\end{array}$ & $\begin{array}{l}T_{\mathrm{m}} \\
\left({ }^{\circ} \mathrm{C}\right)\end{array}$ \\
\hline Blank DPPC & 8.43 & 0.1963 & 42.96 \\
\hline $\mathrm{DPPC}+2 \mathrm{mM} \mathrm{DOX}$ & 11.95 & 0.2856 & 41.86 \\
\hline $\mathrm{DPPC}+2 \mathrm{mM} \mathrm{RAL}$ & 12.12 & 0.2463 & 49.24 \\
\hline $\mathrm{DPPC}+\mathrm{P} 84$ & 8.050 & 0.1928 & 41.78 \\
\hline DPPC+2 mM RAL+P84 & 11.03 & 0.2248 & 49.08 \\
\hline $\begin{array}{l}\mathrm{DPPC}+0.5 \mathrm{mM} \\
\mathrm{DOX}+0.5 \mathrm{mM} \text { RAL }+\mathrm{P} 84\end{array}$ & 12.62 & 0.2752 & 45.88 \\
\hline DPPC+0.5 mM RAL+P84 & 11.51 & 0.2590 & 44.45 \\
\hline $\mathrm{DPPC}+0.5 \mathrm{mM}$ RAL & 10.98 & 0.2312 & 47.50 \\
\hline $\begin{array}{l}\text { DPPC }+0.5 \mathrm{mM} \\
\text { DOX+0.5 mM RAL+ } \\
\text { P84+10,000:1 MNPs }\end{array}$ & 07.89 & 0.2024 & 39.03 \\
\hline $\begin{array}{l}\text { DPPC+0.5 mM } \\
\text { DOX+0.5 mM } \\
\text { RAL+P84+10,000:1 } \\
\text { MNP's }\end{array}$ & 05.63 & 0.1364 & 41.28 \\
\hline
\end{tabular}

$D P P C$ dipalmitoylphosphatidyl choline, DOX doxycycline $\mathrm{HCl}, R A L$ raloxifene $\mathrm{HCl}, M N P s$ magnetic nanoparticles from the liposomes, most likely due to passive diffusion driven by a concentration gradient across the vesicular lipid bilayer. About $1200 \mathrm{nM}$ of DOX was released after $24 \mathrm{~h}$ of dissolution. In contrast, RAL, localized within the lipid bilayers, showed minimal release after $24 \mathrm{~h}$ (Fig. 1). MNP-containing liposomes did not show any drug leakage in the absence of the application of an external magnetic field. However, when subjected to a 30-min exposure to an external electromagnetic field and then stored at $37^{\circ} \mathrm{C}$, a release pattern was observed. As seen in Fig. 6, the samples were collected at various time points $(1,3$, $4,20,21$, and $24 \mathrm{~h}$ ). Before the collection of each sample, the formulations were subjected to $30 \mathrm{~min}$ of radio frequency (RF) exposure and stored at $37^{\circ} \mathrm{C}$ for another $30 \mathrm{~min}$. DOX release was independent of time and observed only after exposure to RF radiation. No RAL release was noted, even under the influence of RF heating.

\section{DISCUSSION}

DOX, a hydrophilic tertiary amine ( $\mathrm{pKa}$ 9.3) with a high polar surface area that is primarily protonated and charged at physiologic $\mathrm{pH}$, and RAL, a relatively hydrophobic drug with large domains of hydrophobicity including an aromatic heterocycle and an aliphatic chain, were incorporated into different compartments (hydrophilic core and lipid bilayers, respectively) of the liposomes. The chemical structures of each of the drugs are shown in Fig. 7. DOX has a high aqueous solubility and permeability and hence belongs to biopharmaceutical classification system (BCS) class I, whereas RAL belongs to BCS class II drugs which consist of low solubility/ high permeability compounds and has a reported log P of 5.7 (26). This may partly explain the high affinity of RAL for the lipid bilayers as compared to DOX.

The inclusion of DOX into DPPC vesicles did not significantly alter the $T_{\mathrm{m}}$ of the liposomes (Fig. 4). This suggests that DOX was not associated with the bilayers and was instead 


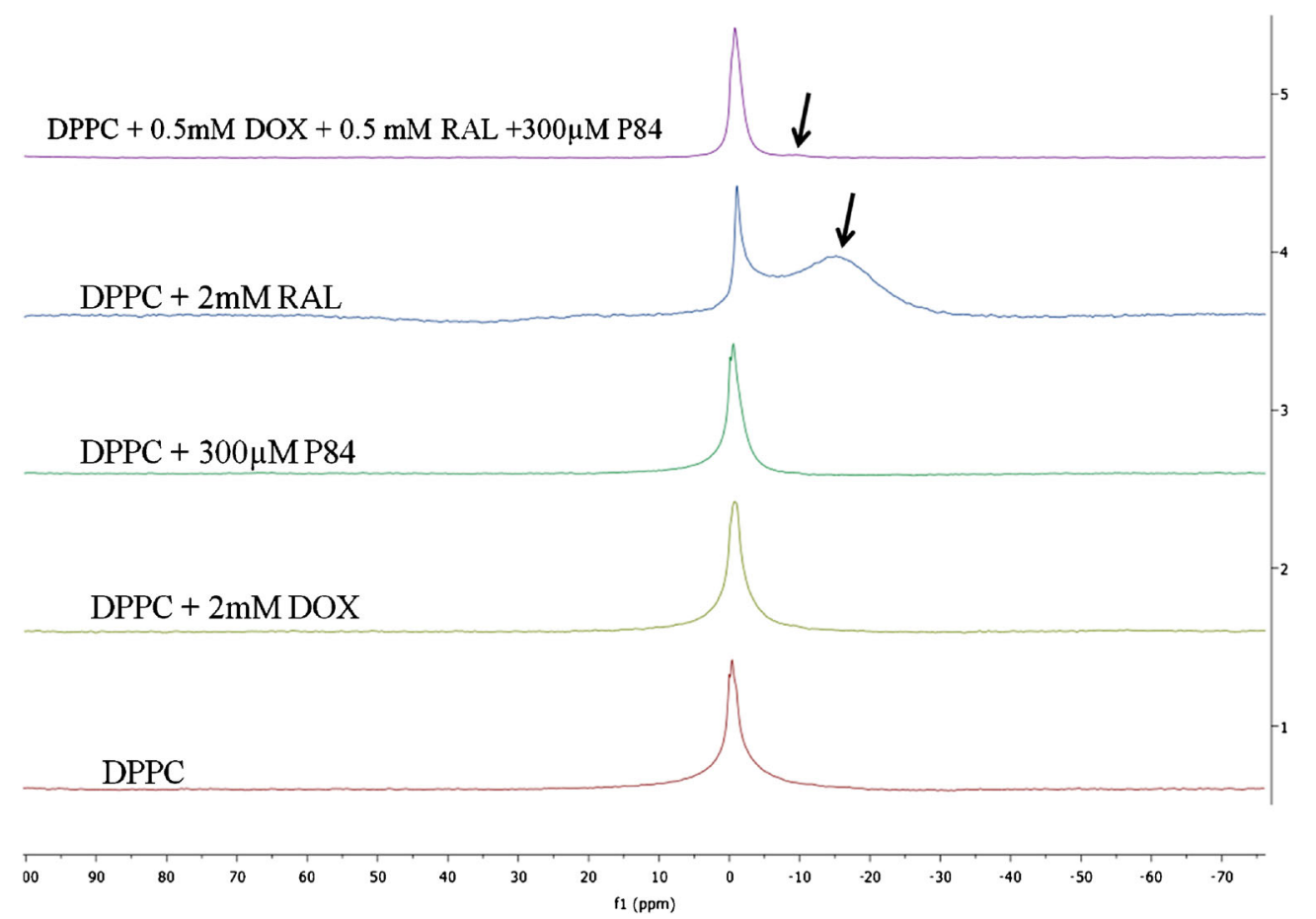

Fig. 5. ${ }^{31} \mathrm{P}-\mathrm{NMR}$ spectra after $60 \mathrm{~K}$ scans for liposomes containing (1) Blank DPPC, (2) DPPC and $2 \mathrm{mM}$ DOX, and (3) DPPC and $300 \mu \mathrm{M}$ P84, (4) DPPC and $2 \mathrm{mM} \mathrm{RAL}$, and (5) DPPC with $0.5 \mathrm{mM}$ RAL and DOX and $300 \mu \mathrm{M}$ P84 dispersed in a 1:9 $\left(\mathrm{D}_{2} \mathrm{O}: \mathrm{H}_{2} \mathrm{O}\right)$ solvent equilibrated at $37^{\circ} \mathrm{C}$. No shielding effect was detected for DOX or P84 containing liposomes. Black arrows indicate the shift in the upward field due to shielding effect of the aromatic rings present in RAL

incorporated in the aqueous core or partly adsorbed onto the bilayer surface. Although the polypropylene oxide (PPO) moieties of P84 were hypothesized to be present in the bilayers (27), the inclusion of P84 did not affect the $T_{\mathrm{m}}$ of DPPC vesicles. However, peak broadening was detected, suggesting a decrease in the cooperativity of the phase transition, as has

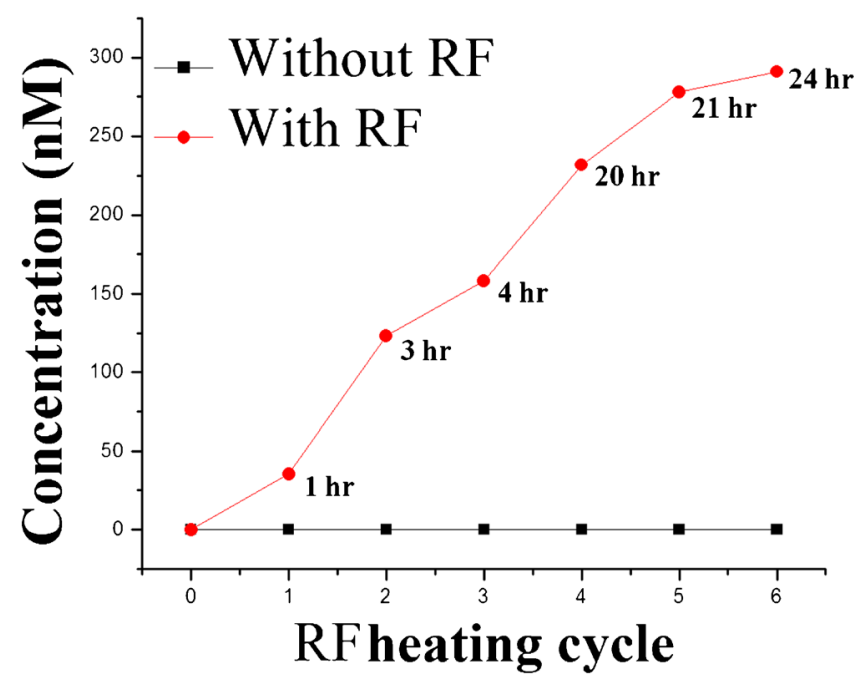

Fig. 6. RF-induced release of DOX from liposomes at $37^{\circ} \mathrm{C}$ and at $\mathrm{pH}$ 7.4 in $137 \mathrm{mM}$ PBS. Both DOX and RAL were encapsulated at 0.5$\mathrm{mM}$ concentration. The lipid/MNP ratio was 10,000:1 that produced local hyperthermia and triggered the release of DOX. The red curve represents the drug release profile as a function of the RF heating cycle and the time points at which samples were collected in real time been reported with the addition of cholesterol (28). This peak broadening effect was only demonstrated in the presence of P84 and/or MNPs that are coated with hydrophobic oleic acid. This is attributed to inclusion of the PPO or oleic acid chains of the MNPs into the lipid bilayers. The RAL-induced shift to a higher $T_{\mathrm{m}}$ was directly proportional to the concentration of RAL included in the liposome formulations. The higher $T_{\mathrm{m}}$ suggests more thermodynamic and mechanical stability of the liposomes. Various studies have revealed that an increase in mechanical stability is associated with an increase in bilayer rigidity and, in turn, colloidal stability (29). Thus, RALcontaining liposomes were more stable than analogous, DOX-containing liposomes. The MNP-induced change in the $T_{\mathrm{m}}$ was inversely proportional to the concentration of MNPs incorporated into the vesicular systems $\left(T_{\mathrm{m}}=41.28^{\circ} \mathrm{C}\right.$ for $\mathrm{L} / \mathrm{N}$ ratio of 20,000/1). This is perhaps due to the hydrophobic interaction of the oleic acid coating on the MNPs with the lipid chains in the vesicle bilayer, thereby forming a less rigid

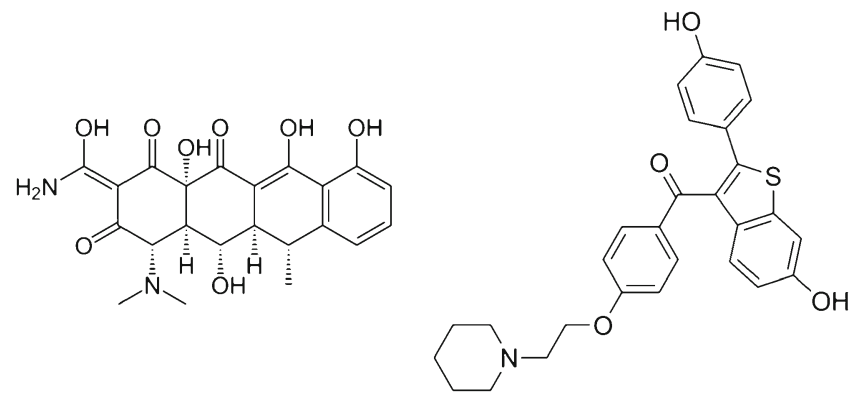

Fig. 7. Chemical structures of DOX (left) and RAL (right) 
lipid bilayer. Although the apparent reduction in bilayer rigidity might theoretically have led to leaky liposomes, drug release studies did not show any leakage from the MNPcontaining liposomes in the absence of radio frequency (RF)-induced heating $(25,30)$.

31 P-NMR analysis revealed that the upfield resonance due to the addition of RAL was likely a result of additional shielding due to the rigid aromatic rings of RAL which oriented themselves in the lipid bilayers in the proximity of the phosphate head groups (Fig. 5). The magnitude of this shielding effect was directly proportional to the concentration of RAL incorporated into DPPC liposomes (31).

Due to stability issues when using a combination of DOX and RAL in the same liposomal system, the concentration of each drug was reduced to $1 \mathrm{mM}$. This system was still unstable, as was an analogous system containing $0.75 \mathrm{mM}$ of each drug, perhaps due to the adsorption of protonated, positively charged DOX ( $\mathrm{pKa}$ 9.3) near the surface phosphate head groups, thereby reducing electrostatic repulsion and leading to aggregation. The concentration of the drug combination used was further reduced to $0.5 \mathrm{mM}$ each. This formulation still exhibited increased aggregation as compared to singledrug loaded liposomes. The reduction in drug concentration did not improve liposome stability, and since a goal of the project was to make stabilized nanoliposomes, PLs were added to the bilayers in order to enhance vesicular stability (14). Pluronics ${ }^{\circledR}$ F-127 and F-108 have been shown to increase the mechanical stability of lipid-based vesicular systems, such as dioleoylphosphatidylethanolamine liposomes (23). Therefore, various grades of PLs were incorporated at different weight percent into the liposomal bilayers in order to stabilize the liposomes containing $0.5 \mathrm{mM}$ of both DOX and RAL, respectively (Table I). Among the four grades of PL that were assessed as liposome stabilizers, only P84 enhanced vesicular stability. Since P84 has the lowest hydrophilic/ hydrophobic (PEO/PPO) ratio, the hydrophobic PPO chains of this particular polymer grade may have undergone the most pronounced insertion into the hydrophobic bilayers of the liposomes, thereby resulting in mechanical stabilization. With the increase in the hydrophilic moiety of the copolymer polyethylene oxide (PEO), there is the possibility of "squeezing out" of the copolymer from the lipid bilayers. With P84 at 1:10 (PL/DPPC) $w / w$ ratio, the optimum amount of hydrophilic PEO chains on the surface and PPO chains inserted into the bilayers of each liposome likely improved vesicular stability due to steric hindrance (32).

This stable system was used for release studies, and no drug was detected in the dialysis or drug release media after $24 \mathrm{~h}$, suggesting complete drug encapsulation. It might be noted that these liposomal formulations displayed minimal leakage of the entrapped material, suggesting the potential advantage of minimal toxicity in vivo when using analogous formulations to entrap potent or toxic drugs with a narrow therapeutic index. In the interest of developing useful drug delivery systems, and in light of the complete absence of drug release from these two-drug containing formulations, it was hypothesized that a trigger mechanism was needed so that the drugs would eventually be released from these liposomes. Accordingly, MNPs that could be subjected to an external electromagnetic field (due to their paramagnetic property), thereby inducing vibration and heating within the lipid bilayer, were successfully incorporated within the liposomal bilayers. Upon exposure to this external electromagnetic field, the MNPs would be expected to produce a local hyperthermia within the bilayers, thereby increasing the temperature above the $T_{\mathrm{m}}$ and triggering drug release. The rate and extent of DOX release from the liposomes triggered by its exposure to an electromagnetic field is shown in Fig. 6. In contrast, electromagnetic irradiation had no effect on RAL release from lipid vesicles. RAL, being highly hydrophobic in nature and strongly interacting with the lipid bilayer as demonstrated by NMR and nano-DSC studies, did not show any release in vitro.

\section{CONCLUSIONS}

In the current investigation, two physicochemically disparate, therapeutically complementary drugs were successfully incorporated into liposomes. This type of combination drug carrier might be used in the treatment of various conditions that may require multiple drug administration in a controlled manner for a synergistic effect, thereby improving therapy. Poor stability is a common observation with liposomal systems, which has been addressed here by the use of PL-induced steric hindrance that maintained a homogenous particle size and improved formulation stability for an enhanced period of time. Notably, RAL had a pronounced effect on liposomal stability, which suggests the possibility of assessing RAL or related compounds for stabilizing otherwise unstable liposomes. Liposomal stabilization was limited by and highly dependent upon the concentration of the incorporated two-drug combination. The presence of PLs, MNPs, and RAL in the bilayers all had a pronounced effect on drug release. Under normal physiological conditions, minimum cargo leakage would be highly desirable as it might reduce systemic exposure and toxicity and promote drug targeting. Embedding MNPs in the bilayers may offer the advantage of controlling and triggering drug release with the aid of a physiologically compatible, noninvasive magnetic field. RAL, and perhaps related compounds, interacts avidly with phospholipid vesicles, thereby apparently conferring additional vesicle stability but compromising RAL release from these systems. The effects of RAL on liposome characteristics and drug release performance underscore the importance of appropriate physical-chemical analysis in order to identify and characterize drug-lipid interactions that may profoundly affect liposome characteristics and performance early in the formulation development process. Although beyond the scope of this work, additional studies that characterize these interactions and their effects may be useful for the design of controlled delivery systems for RAL and related compounds.

\section{ACKNOWLEDGMENTS}

The authors would like to thank Dr. Richard Kingsley for performing the cryo-TEM imaging and EDS scans.

This material is based upon work conducted at a research facility at the University of Rhode Island supported in part by the National Science Foundation EPSCoR Cooperative Agreement \#EPS-1004057. 


\section{REFERENCES}

1. Burra M, Raju Jukanti R, Janga KY, Sunkavalli S, Velpula A, Ampati S, et al. Enhanced intestinal absorption and bioavailability of raloxifene hydrochloride via lyophilized solid lipid nanoparticles. Adv Powder Tech. 2013;24:393-402.

2. Tran TH, Poudel BK, Marasini N, Chi SC, Choi HG, Yong CS, et al. Preparation and evaluation of raloxifene-loaded solid dispersion nanoparticle by spray-drying technique without an organic solvent. Int J Pharm. 2013;443:50-7.

3. Mahmood S, Taher M, Mandal UK. Experimental design and optimization of raloxifene hydrochloride loaded nanotransfersomes for transdermal application. Int J Nanomedicine. 2014;9:4331-46.

4. Smith CJ, Sayles H, Mikuls TR, Michaud K. Minocycline and doxycycline therapy in community patients with rheumatoid arthritis: prescribing patterns, patient-level determinants of use, and patient-reported side effects. Arthritis Res Ther. 2011;13:R168.

5. Aw MS, Khalid KA, Gulati K, Atkins GJ, Pivonka P, Findlay $\mathrm{DM}$, et al. Characterization of drug-release kinetics in trabecular bone from titania nanotube implants. Int J Nanomed. 2012;7:4883-92.

6. Nair M, Krishnan A. Antibiotic releasing biodegradable scaffolds for osteomyelitis. Curr Drug Deliv. 2014;11:687-700.

7. Lianand T, Ho RJ. Trends and developments in liposome drug delivery systems. J Pharm Sci. 2001;90:667-80.

8. Randlesand EG, Bergethon PR. A photodependent switch of liposome stability and permeability. Langmuir. 2013;29:1490-7.

9. Yaroslavov AA, Rakhnyanskaya AA, Yaroslavova EG, Efimova AA, Menger FM. Polyelectrolyte-coated liposomes: stabilization of the interfacial complexes. Adv Colloid Int Sci. 2008;142:43-52.

10. Sabin J, Prieto G, Ruso JM, Hidalgo-Alvarez R, Sarmiento F. Size and stability of liposomes: a possible role of hydration and osmotic forces. Eur Phys J E Soft Matter. 2006;20:401-8.

11. Liang X, Mao G, Ng KY. Mechanical properties and stability measurement of cholesterol-containing liposome on mica by atomic force microscopy. J Colloid Interface Sci. 2004;278:53-62.

12. Chapmanand D, Morrison A. Physical studies of phospholipids. IV. High resolution nuclear magnetic resonance spectra of phospholipids and related substances. J Biol Chem. 1966;241:5044-52.

13. Stark B, Pabst G, Prassl R. Long-term stability of sterically stabilized liposomes by freezing and freeze-drying: effects of cryoprotectants on structure. Eur J Pharm Sci. 2010;41:546-55.

14. Liang X, Mao G, Ng KY. Effect of chain lengths of PEO-PPOPEO on small unilamellar liposome morphology and stability: an AFM investigation. J Colloid Interface Sci. 2005;285:360-72.

15. Wuand G, Lee KY. Interaction of poloxamers with liposomes: an isothermal titration calorimetry study. J Phys Chem B. 2009;113:15522-31.

16. Zhao Y, Alakhova DY, Kim JO, Bronich TK, Kabanov AV. A simple way to enhance Doxil (R) therapy: drug release from liposomes at the tumor site by amphiphilic block copolymer. J Control Release. 2013;168:61-9.

17. Nakano M, Kamo T, Sugita A, Handa T. Detection of bilayer packing stress and its release in lamellar-cubic phase transition by time-resolved fluorescence anisotropy. J Phys Chem B. 2005; 109:4754-60.

18. Yang T, Cui FD, Choi MK, Cho JW, Chung SJ, Shim CK, et al. Enhanced solubility and stability of PEGylated liposomal paclitaxel: in vitro and in vivo evaluation. Int $\mathbf{J}$ Pharm. 2007;338:317-26.

19. Ding X, Cai K, Luo Z, Li J, Hu Y, Shen X. Biocompatible magnetic liposomes for temperature triggered drug delivery. Nanoscale. 2012;4:6289-92.

20. Oliveira H, Perez-Andres E, Thevenot J, Sandre O, Berra E, Lecommandoux $\mathrm{S}$. Magnetic field triggered drug release from polymersomes for cancer therapeutics. J Control Release. 2013;169:165-70.

21. Schleich N, Sibret P, Danhier P, Ucakar B, Laurent S, Muller RN, et al. Dual anticancer drug/superparamagnetic iron oxide-loaded PLGA-based nanoparticles for cancer therapy and magnetic resonance imaging. Int J Pharm. 2013;447:94-101.

22. Chen Y, Bose A, Bothun GD. Controlled release from bilayerdecorated magnetoliposomes via electromagnetic heating. ACS Nano. 2010;4:3215-21.

23. Bergstrand EK. Effects of poly (ethylene oxide)-poly (propylene oxide)-poly (ethylene oxide) triblock copolymers on structure and stability of liposomal dioleoylphosphatidylethanolamine. J Colloid Interface Sci. 2004:276:400-7.

24. Quinn PJ. The effect of tocopherol on the structure and permeability of phosphatidylcholine liposomes. J Contr Release. 2012;160:158-63.

25. Cullisand B, de Kruijff PR. Lipid polymorphism and the functional roles of lipids in biological membranes. Biochim Biophys Acta. 1979;559:399-420.

26. Tran TH, Poudel BK, Marasini N, Woo JS, Choi HG, Yong CS, et al. Development of raloxifene-solid dispersion with improved oral bioavailability via spray-drying technique. Arch Pharm Res. 2013;36:86-93.

27. Krylova TV, Melik-Nubarov NS. Effects of block copolymers of alkylene oxides on the permeability of lipid membranes: possible origins of biological activity. Dokl Chem. 2001;380:267-70.

28. Bolean M, Simao AM, Favarin BZ, Millan JL, Ciancaglini P. The effect of cholesterol on the reconstitution of alkaline phosphatase into liposomes. Biophys Chem. 2010;152:74-9.

29. Anderson M, Omri A. The effect of different lipid components on the in vitro stability and release kinetics of liposome formulations. Drug Deliv. 2014;11:33-9.

30. Chen Y, Xiao D, Bose A, Deng R, Bothun GD. Low-dose chemotherapy of hepatocellular carcinoma through triggered-release from bilayer-decorated magnetoliposomes. Colloids Surf B: Biointerfaces. 2014;116:452-8.

31. Amicangelo JC, Leenstra WR. Synthesis, characterization, and interlayer distance study of zirconium phosphonates with stoichiometric variation of methyl and $p$-aminobenzyl pendant groups. Inorg Chem. 1998;37:5317-23.

32. Markus Johnsson MS, Karlsson G, Edwards K. Effect of PEO-PPO-PEO triblock copolymers on structure and stability of phosphatidylcholine liposomes. Langmuir. 1990;15:6314-25. 